\title{
Sur quelles connaissances se baser pour évaluer l'état de santé des populations de truite commune (Salmo trutta)?
}

\section{What do we know to evaluate the health of brown trout (Salmo trutta) populations?}

\author{
V. Gouraud( ${ }^{(1)}$, P. Baran ${ }^{(2)}, A$. Bardonnet $^{\left(3,3^{\prime}\right)}$, C. Beaufrère $(4)$, \\ H. Capra ${ }^{(5)}$, A. Caudron $^{(6)}$, M. Delacoste ${ }^{(7)}$, J.M. Lascaux ${ }^{(8)}$,

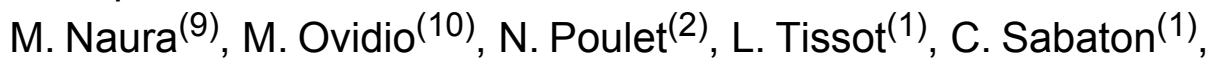
J.-L. Baglinière ${ }^{(11)}$

(1) EDF Recherche et Développement, Laboratoire National d'Hydraulique et Environnement, 6 quai Watier, 78401 Chatou Cedex, France

(2) Onema, Pôle Écohydraulique, avenue professeur Camille Soula, 31400 Toulouse, France

(3) INRA, UMR Ecobiop, Aquapôle, 64310 St Pée sur Nivelle, France

(3') UPPA, UMR Ecobiop, UFR des sciences de la Côte Basque, 64600 Anglet, France

(4) GéoDiag, 8 bis rue Camy, 64000 Pau, France

(5) IRSTEA, Laboratoire Dynam, Unité Milieux Aquatiques, Écologie et Pollutions, 5 rue de la Doua, CS 70077, 69626 Villeurbanne Cedex, France

(6) INRA UMR CARRTEL, Centre Alpin de Recherche sur les Réseaux Trophiques et Écosystèmes Limniques, 75 avenue de Corzent, 74203 Thonon-les-Bains cedex, France

(7) Fédération de Pêche des Hautes Pyrénées, 20 boulevard du 8 mai 1945, 65000 Tarbes, France

(8) ECOGEA 10 avenue de Toulouse, 31860 Pins Justaret, France

(9) University of Southampton, Highfield, Highfield Avenue, SO17 1BJ, Southampton, UK

(10) Université de Liège, Applied and Fundamental Fish Research Center (AAFISH-RC), Unité de Biologie du Comportement, Laboratoire de Démographie des Poissons et d'Hydroécologie, 10 chemin de la Justice, 4500 Tihange, Belgique

(11) INRA-Agrocampus Ouest, Écologie et Santé des Écosystèmes, UMR 985, 65 rue de St Brieuc, CS 84215 , 35042 Rennes Cedex, France

Résumé - Le retour au premier plan de la notion de santé des écosystèmes met en évidence la préoccupation de la société à mettre en œuvre des mesures pour la protection des milieux anthropisés. Les critères d'évaluation de la santé des peuplements piscicoles mis en œuvre sont rarement débattus au sein de la communauté scientifique. L'exercice proposé ici devait permettre d'avoir ce débat sur la truite commune (Salmo trutta), espèce phare des cours d'eau situés en tête de bassin, qui représentent la majorité du réseau hydrographique français. La démarche visait à recueillir la conception qu'avait un nombre restreint d'experts, sur le fonctionnement de ces populations et sur les critères d'évaluation de leur fonctionnement. Les paramètres clé régulant chaque stade de développement ont été identifiés et hiérarchisés. Un consensus s'est dégagé sur le fait que, dans l'état actuel des connaissances, le diagnostic 
peut être établi sur la base de l'analyse des paramètres abiotiques essentiels pour la biologie et, plus difficilement, sur l'analyse des paramètres biotiques. Pour tous les stades de développement, les paramètres identifiés sont liés à l'habitat (qualité du substrat, conditions hydrauliques, température et qualité de l'eau), l'hydrologie et la connectivité. Des connaissances demandent d'être acquises pour définir des critères biologiques. Cela implique de renforcer la surveillance biologique à long terme et les actions de recherche pour caractériser la variabilité des paramètres biologiques et identifier les échelles spatio-temporelles pertinentes et les processus fonctionnels.

Mots-clés - truite commune, critères d'évaluation, experts, diagnostic, paramètres clé

Abstract - The renewed emphasis on the concept of ecosystem health highlights society's interest in taking measures to protect environments affected by human activity. The criteria used for evaluating fish population health are rarely discussed within the scientific community. The present study attempts to discuss these for brown trout (Salmo trutta), a flagship freshwater species typical of headwater streams which represent most of the French hydrographic network. This initiative aimed at gathering ideas from a limited number of experts on the criteria for evaluating trout population health. The main key parameters were identified and organized into a hierarchical framework for each development stage. A consensus emerged on the fact that at the current state of knowledge, a diagnosis can be established based on the analysis of essential abiotic parameters but with more difficulty on the analysis of biotic parameters. For all development stages, the identified parameters are linked to habitat (substrate, hydraulic parameters, temperature and water quality), hydrology and connectivity. Further knowledge is required to identify relevant biological criteria. This implies long-term biological monitoring and research programs to better understand and identify biological parameters and their variability as well as relevant spatiotemporal scales and functional processes.

Key words - brown trout, assessment criteria, expert assessment, diagnosis, key parameters

\section{INTRODUCTION}

Dans le contexte de l'influence grandissante du changement global et des pressions anthropiques de ce début de $21^{\mathrm{e}}$ siècle, la protection du milieu, la gestion des ressources en eau et des ressources biologiques naturelles sont au centre des enjeux sociétaux et scientifiques. Les besoins de la société sur la compréhension du fonctionnement écologique des écosystèmes aquatiques se sont renforcés d'autant qu'ils font apparaître la liaison environnement-santé. Cette notion de santé per- mettant habituellement de caractériser la vitalité des individus et des populations chez l'homme et la faune domestique et sauvage, a été étendue à celle des écosystèmes. Elle a été développée au cours de la décennie 90 (Rapport et al., 1998) et peut être évaluée et se mesurer en termes i) d'organisation correspondant à la diversité et au nombre d'interactions entre les différentes composantes du système, ii) de résilience ${ }^{1}$, iii) de vigueur mesurée

(1) En écologie, elle est assimilée à la récupération après un stress traumatique comme peut l'être un forçage anthropique. 
en termes d'activité ou de productivité primaire. Ce retour au premier plan de la notion de santé des écosystèmes met en évidence la préoccupation de la société à mettre en œuvre des mesures de protection pour les écosystèmes influencés par l'activité humaine.

Pour ce faire, la recherche et le développement d'outils scientifiques et d'outils de régulation publique demandent à être déployés : d'une part, pour disposer d'outils d'évaluation et de compréhension du fonctionnement des écosystèmes aquatiques en intégrant la notion d'incertitude inhérente à tout phénomène biologique; d'autre part, pour faciliter le dialogue entre les acteurs sur la question de l'usage des écosystèmes. De fait, ces deux types d'éléments relatifs aux connaissances écologiques et à leur application en lien avec les besoins de la société sont à considérer comme distincts mais complémentaires (Lévêque, 2001). Ils relèvent respectivement de l'écologie et des sciences de l'environnement. La mise en œuvre de toute réglementation environnementale demande de faire appel à ces deux types de connaissances.

La Directive cadre européenne sur l'eau (DCE ; UE 2000) fixe notamment comme objectif l'atteinte du bon état écologique (ou bon potentiel pour les masses d'eau fortement modifiées ou artificielles) des différents compartiments de l'écosystème aquatique (poissons, invertébrés, végétaux) à l'horizon 2015, avec obligation de résultats pour tous les milieux aquatiques naturels. Ce passage d'objectifs de moyens à des objectifs de résultats place les communautés biologiques, avec les paramètres physico-chimiques, comme juges de paix (Roche et al., 2005). Le caractère simple et synthétique de ce dispositif le fait s'inscrire dans une vague de politiques publiques émergeant dans le monde depuis les années 90 et désignées sous le vocable «policy design » (Lascoumes \& Le Gales, 2005). La manière dont les connaissances écologiques sont utilisées pour permettre la réalisation de ces objectifs relève clairement du domaine des sciences de l'environnement.

En revanche, la base de connaissances sur laquelle la DCE doit s'appuyer pour décrire le fonctionnement des écosystèmes fait appel au domaine de la biologie des populations et de l'écologie des communautés. Plus précisément, l'application de cette directive demande de disposer d'outils de diagnostic d'état et du fonctionnement (évolution) des communautés biologiques. Dans ce cadre, des indicateurs sont développés ou sont en cours de développement par différentes unités de recherche pour les poissons (Pont et al., 2006), les invertébrés (Mondy et al., 2012) et les végétaux (Haury et al., 2006). Ils se basent sur les données biologiques et écologiques disponibles à large échelle et le développement de modèles quantitatifs pour évaluer le type et le niveau de perturbations des écosystèmes aquatiques. Cherchant à distinguer de façon standardisée les états critiques des situations satisfaisantes, ils ont pour vocation d'offrir la possibilité à des acteurs différents (scientifiques, gestionnaires, politiques et citoyens) de dialoguer entre eux. Ils se doivent de constituer ainsi des éléments d'aide à la décision tout en permettant une prise 
de conscience lorsque des anomalies sont détectées.

Si ces indicateurs biologiques sont intéressants pour des comparaisons inter-sites, des critères demeurent nécessaires pour évaluer finement à l'échelle d'un site le fonctionnement de l'écosystème. Les critères d'évaluation utilisés au sein de la communauté scientifique sont, de plus, rarement débattus. L'exercice proposé ici vise à avoir ce débat sur les cours d'eau situés en tête de bassin ou de faible ordre de drainage qui représentent la majorité du réseau hydrographique français. Ces parties de cours d'eau sont des milieux fragiles, à forte hétérogénéité spatiale et soumis à diverses pressions anthropiques. Quatre-vingt pourcents des ouvrages hydroélectriques sont notamment implantés en tête de bassin. Dans le cadre de travaux agricoles, des rectifications et des remblaiements des petits chevelus sont souvent observés. Des questions de diagnostic de fonctionnement se posent donc fréquemment sur ce type d'écosystème. Ces questions portent le plus souvent sur la truite commune (Salmo trutta) qui est une espèce phare (porte drapeau) prépondérante dans le peuplement pisciaire sur cette zone du cours d'eau et qui a une forte valeur patrimoniale et halieutique.

De nombreuses connaissances ont été acquises sur la truite commune par rapport aux autres espèces pisciaires. Sa biologie et son écologie ont été largement décrites dans la littérature. Des outils opérationnels ont été développés pour apprécier les besoins en termes d'habitat physique pour les différents stades de développement. Ces connaissances nécessitent d'être architecturées en vue d'être à même de dresser un diagnostic sur le fonctionnement de ces populations.

Cette démarche s'inscrit dans ce contexte et propose de recueillir l'avis d'un nombre restreint d'experts, avec pour objectif de répondre aux deux questions suivantes:

- quelles sont les connaissances dont nous disposons pour évaluer le fonctionnement des populations de truite?

- peut-on établir des critères d'évaluation du fonctionnement de ces populations?

\section{MATÉRIELS ET MÉTHODES}

\subsection{Description des étapes de la démarche}

Une quinzaine d'experts a été rassemblée au cours d'un séminaire de trois jours dans l'objectif de statuer sur les processus et les critères d'évaluation du fonctionnement des populations de truite. Les experts ont été amenés à se prononcer sur les processus agissant sur ces populations à différentes échelles spatio-temporelles. Un emboîtement des échelles physiques du bassin jusqu'aux micro-habitats a été considéré. Pour chacune des étapes suivantes, une synthèse des principaux processus connus a été réalisée par un des experts pour initier les échanges.

Étape 1: Description à large échelle du milieu physique et des processus hydromorphologiques

- Le $1^{\text {er }}$ débat a porté sur la caractérisation du type de zone sur lequel 
les critères d'évaluation des populations de truite seront susceptibles de s'appliquer. Un essai pour caractériser de manière quantitative ce type de zone a été réalisé afin de préciser au préalable la zone d'application des critères d'évaluation des populations de truite que nous souhaitions faire émerger. La réflexion a porté sur les caractéristiques permettant d'évaluer le potentiel d'une zone et a ciblé, par conséquent, les paramètres peu modifiés par les activités anthropiques.

- Le $2^{\mathrm{e}}$ débat a porté sur la description des processus hydromorphologiques de contrôle des paramètres du milieu à l'aide de la construction de schémas. Un schéma général cherchant à retranscrire les paramètres hydromorphologiques concernés a été construit collectivement. Étaient visés ici les processus hydromorphologiques qui soutiennent les communautés biologiques, les altérations anthropiques et les critères physiques majeurs qui créent l'habitat ainsi que les processus qui les génèrent.

Étape 2 : Description des processus de fonctionnement des populations de truite via l'établissement des relations entre les populations de truite et les différents paramètres du milieu

- Dans un premier temps, les échanges ont visé à s'accorder sur une description simplifiée du cycle biologique de la truite qui peut présenter une grande variance selon le type de milieu. L'objectif était ici de restituer la vision que les experts se sont forgés de ce cycle, vision acquise à partir d'études ponctuelles menées par les participants eux-mêmes ou acquises à travers la littérature, ceci sur des sites aux caractéristiques physiques distinctes et aux populations dissemblables. Bien qu'imparfaite, cette vision avait pour objectif de fixer des gammes de valeurs moyennes pouvant être appliquées aux populations des cours d'eau français.

- Dans un second temps, l'exercice a porté sur l'identification des facteurs influant sur les stades de développement. L'objectif ici n'était pas d'établir un recensement des facteurs limitants cités dans la littérature et observés en milieu naturel mais de rassembler et de confronter les connaissances des experts concernant les paramètres influant sur les stades de développement de la truite afin d'aboutir à un consensus sur les paramètres prépondérants. Pour ce faire, des schémas ont été élaborés par stade de développement en distinguant les paramètres favorables et les paramètres limitants. Les gammes de valeur de ces paramètres sont précisées lorsque l'état des connaissances est jugé bon.

Étape 3: Hiérarchisation des critères d'évaluation clés du fonctionnement des populations

- À partir de la représentation des processus effectuée au préalable, le but était de hiérarchiser les processus mis en œuvre et de définir les critères d'évaluation de ces processus (évaluation directe ou indirecte par la mesure de paramètres). 


\subsection{Choix des experts}

Les critères retenus pour le choix des experts ont été :

- des scientifiques travaillant, soit pour des organismes de recherche, soit pour des organismes gestionnaires, l'objectif étant de disposer des différentes approches d'évaluation du milieu susceptibles d'émaner de ces deux types d'acteurs ;

- des personnes disposant de connaissances leur permettant d'évaluer le fonctionnement de l'écosystème aquatique et d'identifier les causes d'altération (c'est-à-dire de faire le lien avec les processus physiques agissant sur les communautés biologiques). Plus de la moitié des experts réunis avaient soutenu une thèse sur l'écologie de la truite commune ;

- des personnes ayant au moins 10 ans d'expérience et étant francophones pour éviter dans un premier temps tout problème de barrière dû à la langue.

L'expertise et la diversité des sujets d'intérêt et des expériences ont été également un critère du choix des experts. L'objectif était de recueillir la conception qu'ont les experts des paramètres influant sur les populations de truite, conception intégrant a priori leurs propres travaux et leurs connaissances des travaux issus de la littérature.

\subsection{Restitution de la démarche}

Les résultats pour chacune de ces étapes sont synthétisés en explicitant le cas échéant les points de convergence et de divergence parmi les experts. Les résultats acquis ne représentent pas un état des lieux exhaustif des connaissances actuelles mais rassemblent les avis et les connaissances d'experts et les fruits de la réflexion collective menée sur cette question complexe de l'évaluation du fonctionnement des populations de truite.

\section{RÉSULTATS}

3.1 Description à large échelle des processus de fonctionnement du milieu physique

3.1.1 Caractérisation de la zone d'application des critères d'évaluation du fonctionnement des populations de truite

Les caractéristiques générales des différentes zones rencontrées de l'amont vers l'aval d'un cours d'eau peuvent être appréhendées à l'aide des classifications et typologies issues de la littérature. Les diverses classifications existantes sont basées, soit sur les conditions morphologiques, soit sur les communautés biologiques, soit sur une combinaison des deux (Botosaneanu, 1988 ; Frissell et al., 1986 ; Huet, 1949 ; Illies \& Botosaneanu, 1963 ; Kondolf et al., 2003 ; Montgomery et al., 1999 ; Ricker, 1934 ; Vannote et al., 1980 ; Verneaux, 1973). La validité des espèces piscicoles prédites par ces classifications a suscité le débat. La confrontation des zonations piscicoles fournies par ces classifications avec des observations de terrain met souvent en évidence des divergences. Ceci probablement en raison des multiples facteurs biotiques et abiotiques qui déterminent la distribution des espèces. L'applicabilité de ce type d'outils suppose l'existence de 
zones homologues même très éloignées géographiquement avec des espèces similaires. Plus on s'éloigne des cours d'eau initialement étudiés, plus le système originel doit être modifié pour s'appliquer aux conditions locales (Wasson, 1989).

Les critères utilisés dans ces classifications sont très divers mais on dénote des facteurs communs : pente, distance à la source, taille du bassin versant, largeur, rang de Stralher (1957), température de l'eau. Des travaux sur la modélisation des distributions d'occurrence de la truite en fonction de variables environnementales (pente, taille bassin versant, température de l'eau) montrent la gamme très étendue de la valeur des paramètres retenus (Pont, 2009).

II est ressorti du débat :

- que la succession des différentes zones en rivière - dont la zone à truite - a été approchée par différents travaux de classification et de typologie. Cependant, ces travaux ne permettent pas de caractériser de façon généralisée les paramètres décrivant les différentes zones, ces paramètres restant, soit trop imprécis, soit trop spécifiques des sites sur lesquels se sont fondés ces travaux ;

- le corollaire est qu'il est difficile de statuer sur des valeurs précises de paramètres pour définir si la zone où l'on se situe est susceptible d'être une zone présentant des populations de truite ;

- qu'en pratique, la comparaison, pour un site donné, des résultats issus des différentes classifications/typologies peut, cependant, permettre de cerner de manière très globale le peuplement attendu (en l'occurrence population de truite ou non);

- que l'espèce a, néanmoins, des exigences écologiques propres incontournables : une faible amplitude thermique, une forte saturation en oxygène dissous, une bonne qualité de l'eau en général (normes de qualité des eaux salmonicoles, cf. Tab. 1) et un accès à des zones favorables à la reproduction (Baglinière, 1999) sont des pré-requis essentiels.

Suite à ce constat, seuls des critères qualitatifs ont pu émerger pour caractériser la zone d'application de la présente réflexion. Il a été choisi de porter la réflexion sur les zones dont le milieu physique permet le développement d'un peuplement dominé en abondance par la truite commune. Ces zones doivent a minima présenter les pré-requis décrits ci-dessus (faible amplitude thermique, forte saturation en oxygène dissous, bonne qualité d'eau) et présenter des critères morphologiques offrant, en l'absence d'altération anthropique, l'ensemble des habitats permettant d'accomplir le cycle biologique de cette espèce (reproduction, grossissement des jeunes stades et croissance des adultes).

\subsubsection{Description des processus hydromorphologiques}

II ressort que la dynamique d'un écosystème fluvial dépend de paramètres abiotiques opérant à large échelle, qui sont liés à la localisation géographique, à savoir le climat, la géologie et le relief. A l'instar de nombreux auteurs (Amoros \& Petts, 1993 ; Bravard \& Petit, 1997 ; 
Tableau I. Normes de qualité d'eau des eaux salmonicoles d'après le journal de la CEE du 14/08/78 et dont les directives ont été adoptées au conseil des Ministres du 30/05/78 (in Baglinière \& Maisse, 1991).

Table I. Water quality standards for salmonid waters from the journal of the EEC of 14 August 1978 and whose guidelines were adopted in the Council of Ministers of 30 May 1978 (in Baglinière \& Maisse, 1991).

\begin{tabular}{|c|c|c|}
\hline Paramètres & Guide & Impérative \\
\hline \multirow[t]{2}{*}{ Température $\left({ }^{\circ} \mathrm{C}\right)$} & & $\begin{array}{c}21,5^{\circ} \mathrm{C}(\bullet) \\
10,0^{\circ} \mathrm{C}(\bullet) \text { pendant la } \\
\text { reproduction }\end{array}$ \\
\hline & \multicolumn{2}{|c|}{$\begin{array}{l}\text { Augmentation en aval d'un rejet: } \\
\qquad 1,5^{\circ} \mathrm{C}(\bullet)\end{array}$} \\
\hline Oxygène dissous $\left(\mathrm{mg}^{\mathrm{L}} \mathrm{L}^{-1} \mathrm{O}_{2}\right)$ & $\begin{array}{c}50 \%>9 \\
100 \%>7\end{array}$ & $\begin{array}{c}50 \%>9 \\
\text { Jamais inférieur } \\
\text { à } 6 \mathrm{mg} \cdot \mathrm{L}^{-1}(\bullet)\end{array}$ \\
\hline $\mathrm{pH}$ & & $6-9(\bullet)$ \\
\hline Matières en suspension $\left(\mathrm{mg} \cdot \mathrm{L}^{-1}\right)$ & $<25(\bullet)$ & \\
\hline $\begin{array}{l}\mathrm{DBO}_{5}\left(\mathrm{mg} \cdot \mathrm{L}^{-1} \mathrm{O}_{2}\right) \\
\text { Nitrites }\left(\mathrm{mg} \cdot \mathrm{L}^{-1} \mathrm{NO}_{2}\right) \\
\text { Ammoniac }\left(\mathrm{mg} \cdot \mathrm{L}^{-1} \mathrm{NH}_{3}\right) \\
\text { Ammonium }\left(\mathrm{mg} \cdot \mathrm{L}^{-1} \mathrm{NH}_{4}\right) \\
\text { Chlore résiduel }\left(\mathrm{mg} \cdot \mathrm{L}^{-1} \mathrm{HCO}\right) \\
\text { Zinc total }\left(\mathrm{mg} \cdot \mathrm{L}^{-1} \mathrm{Zn}\right) \\
\text { Cuivre soluble }\left(\mathrm{mg} \cdot \mathrm{L}^{-1} \mathrm{Cu}\right)\end{array}$ & $\begin{array}{c}<3 \\
<0,01 \\
<0,005 \\
<0,04 \\
<0,04\left(^{*}\right)\end{array}$ & $\begin{array}{l}<0,025 \\
<1(\bullet) \\
<0,005 \\
<0,3(\bullet)\end{array}$ \\
\hline
\end{tabular}

(•) Dérogations possibles

(*) Pour une dureté de l'eau de $100 \mathrm{mg} \cdot \mathrm{L}^{-1} \mathrm{CaCO}_{3}$. Des normes sont établies pour différentes duretés.

Kondolf et al., 2003 ; Lévêque, 2001 ; Rosgen, 1996), on peut proposer une hiérarchie de ces facteurs montrant les relations enchaînées entre les différentes échelles. Ici, ont été ajoutées certaines pressions d'origine anthropique, parmi les plus couramment impliquées agissant à l'échelle régionale, du bassin versant, du tronçon, de la séquence macrohabitat et du microhabitat. Le schéma construit collectivement est retranscrit sur la figure 1.

\subsubsection{1 À l'échelle régionale}

La géologie (notamment nature et répartition des roches mères), et le climat (précipitations et autres facteurs d'érosion), déterminent le relief et son évolution. Ainsi, la forme des vallées, l'organisation du réseau hydrographique, le transfert et la répartition des stocks de sédiments, etc. découlent de l'histoire géologique et de la situation géographique et interviennent directement 


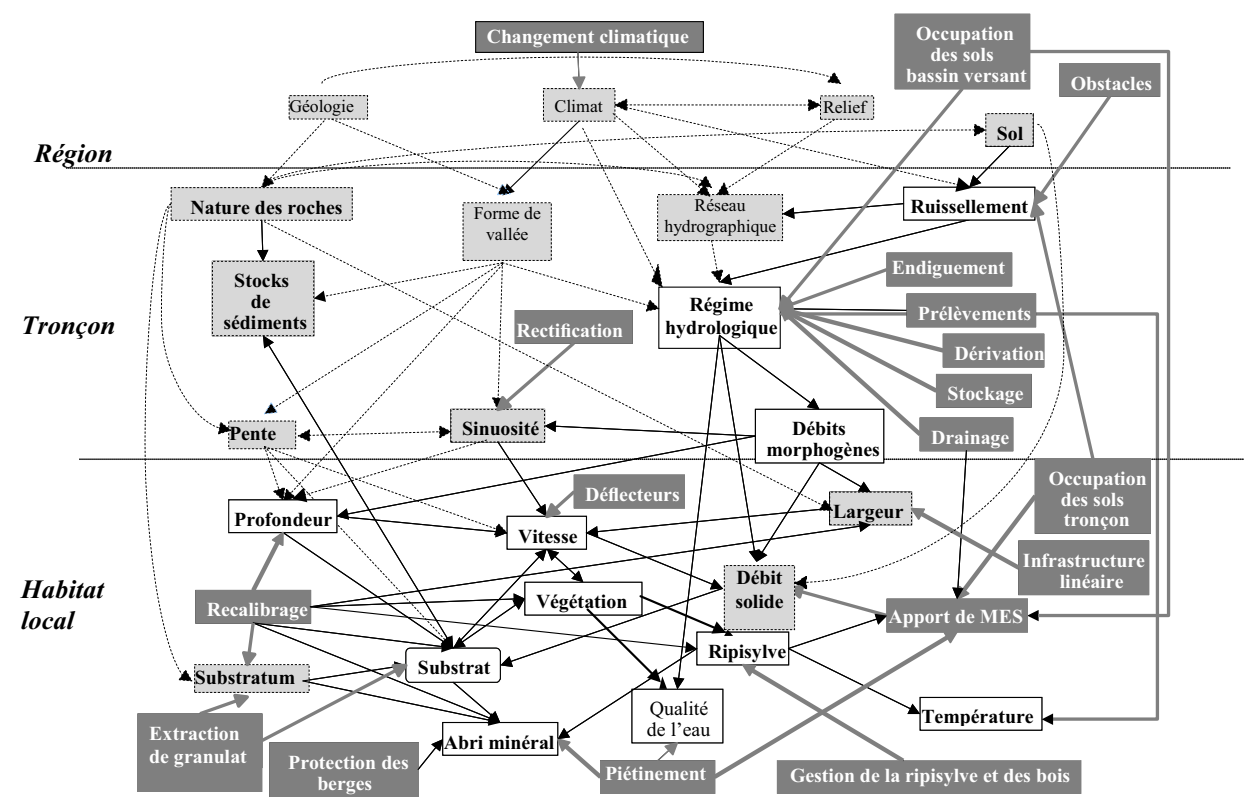

Fig. 1. Description des processus morphologiques et des perturbations liées à des usages anthropiques des cours d'eau et des bassins versants altérant la dynamique fluviale (en gris foncé : les altérations anthropiques, en gris clair : les paramètres abiotiques dont les déterminants opèrent à large échelle ; en blanc : les paramètres abiotiques à l'échelle locale).

Fig. 1. Description of morphological processes and disturbances related to human uses of rivers and watersheds altering river dynamics (in dark grey: anthropogenic changes, in light grey: abiotic parameters of which determinants operate on a large scale; in green: abiotic parameters locally).

sur les flux liquides (débit, écoulement) et solides (alluvions, matières en suspension) des cours d'eau.

\subsubsection{2 À l'échelle du bassin versant}

Les paramètres qui contrôlent le ruissellement, comme le sol et la végétation, conditionnent également ces flux, donc l'organisation et le fonctionnement du réseau hydrographique.

C'est ainsi que les phénomènes d'érosion interne et externe, la dynamique des crues (formation, propagation, etc.) et du transport solide dépendent de l'occupation des sols et des obstacles au ruissellement ou à l'écoulement, non seulement au sein des lits mineur et majeur des cours d'eau mais aussi sur toute la surface du bassin versant considéré.

\subsubsection{3 À l'échelle du tronçon}

On reprendra ici la définition du tronçon donné par Malavoi (1989) qui identifie ce dernier comme une zone où la morphologie générale de la rivière est homogène.

La dynamique spatio-temporelle d'un cours d'eau dépend de son régime hydrologique et sédimentologique, de 
la géomorphologie de la vallée et des compartiments qui caractérisent l'hydrosystème fluvial (lit mineur, lit majeur, espace de mobilité, etc.). La forme de la vallée et la nature des roches qui en tapissent le fond déterminent la sinuosité et la pente longitudinale du cours d'eau. Les débits morphogènes interagissent avec la largeur et la profondeur du lit mineur du cours d'eau et déterminent sa capacité à renouveler ses formes, les milieux et les habitats aquatiques.

Les activités et les aménagements (barrage, drainage, prélèvement, stockage, dérivation, etc.) qui modifient les volumes d'eau s'écoulant dans le réseau hydrographique, dans le temps ou dans l'espace, perturbent le régime hydrologique et peuvent modifier la fréquence des crues morphogènes. Les travaux (curage, extractions, rectifications, endiguements, etc.) qui modifient le gabarit du lit mineur et les connexions avec le lit majeur, influencent les conditions d'écoulement, le transport solide (par charriage de fond ou en suspension) et donc la qualité du substrat.

Ainsi des travaux d'assainissement agricole, combinant curage, recalibrage et endiguement, ont souvent conduit à la disparition du plancher alluvial (sables, galets) et à l'uniformisation de la topographie du fond du lit mineur.

\subsubsection{4 À l'échelle locale}

À l'échelle locale (séquence, faciès ambiance et micro-habitat (Malavoi, 1989), les déterminants clés du fonctionnement des communautés biologiques sont l'habitat physique (profondeur, vitesse, substrat, abri), l'hydroclimat (température et lumière) et les ressources trophiques. La production biologique dépend de la végétation externe (ripisylve), de la production primaire autochtone et du transport de matières organiques depuis l'amont. L'abondance, la stabilité ou le renouvellement des alluvions qui constituent le substrat dépendent des conditions d'écoulement, à l'échelle locale.

Les obstacles transversaux qui freinent ou interrompent les flux liquides et solides, les travaux de curage et de recalibrage qui modifient la profondeur, la largeur, les abris, mais aussi la présence et la densité/continuité de la ripisylve, etc. ont des impacts sur les capacités d'accueil du milieu. Le piétinement des berges par le bétail, en augmentant la charge sédimentaire et en modifiant la qualité de l'eau (notamment la turbidité), constitue également une pression importante à l'échelle de la séquence ou du macrohabitat (notamment pour les frayères) (Massa et al., 2000). Enfin, des apports azotés voire phosphorés peuvent entraîner de nouvelle conditions trophiques induisant une augmentation des conditions de croissance pour le poisson (Rivot et al., 2009).

\subsubsection{Emboîtement des échelles}

$\mathrm{Au}$ sein du bassin versant et du réseau hydrographique fluvial, les multiples paramètres qui déterminent et caractérisent l'état et le fonctionnement hydromorphologique des cours d'eau sont fortement interdépendants et interagissent étroitement. Ils déterminent ainsi un système dynamique complexe sur lequel les activités humaines constituent autant de forces de pressions auxquelles les cours d'eau et les milieux aquatiques associés vont réagir et tenter de s'adapter. 
L'ensemble de ce cadre d'habitat physique détermine les traits biologiques des populations ainsi que leurs adaptations stratégiques au milieu comme le définit la théorie de l'habitat templet (Southwood, 1977, 1988). Les réponses biologiques des espèces qui utilisent cet habitat vont dépendre de sa qualité, de sa dynamique temporelle, de sa répartition spatiale et de la façon dont les différents habitats sont reliés entre eux. Mais elles vont dépendre également de l'expression du pool génique dont les populations de truite sont issues leur permettant de développer des mécanismes adaptatifs via un phénotype ou des traits populationnels.

3.2 Description des processus de fonctionnement des populations de truite

\subsubsection{Représentation simplifiée du cycle biologique de la truite}

Les experts se sont accordés sur une description simplifiée du cycle biologique de la truite, description sur laquelle s'appuyer pour identifier ensuite les facteurs prépondérants. La période de frai retenue s'étale de novembre à février, l'éclosion des œufs entre les mois de janvier et février et l'émergence de mars à avril. La survie de la ponte à l'éclosion est estimée à 0,8 (Hobbs, 1940) avec des seuils létaux pour la température et l'oxygène (Humpesch, 1985 ; Raleigh et al., 1986). Sur les cours d'eau présentant un habitat de bonne qualité (absence de colmatage), la survie de l'éclosion à l'émergence peut à la rigueur être considérée comme constante $(0,85$ sur les deux mois). Après la résorption et l'émergence des frayères, au mois de mars, le phénomène de mortalité densité-dépendance se traduit par une survie maximale d'environ $10 \%$ pour les alevins résorbés passant l'été (Gouraud, 1999). Le taux de survie annuelle des juvéniles de l'année est estimé dans les conditions optimales entre 5 et $7 \%$ tandis que celui des juvéniles $(>0+)$ et des adultes est estimé entre 30 et $50 \%$ (Maisse \& Baglinière, 1999). Dans la majorité des cas, l'espérance de vie est de 4 ans avec des longévités allant jusqu'à 7 ans sur certains sites (voire exceptionnellement 12 ans). La maturation est plus précoce chez les mâles ( 2 ans) que chez les femelles ( 3 ans). Ces dernières ont une fécondité variant de 1000 à 2000 œufs par kg. Le rapport des sexes dans la population est le plus souvent considéré comme équilibré $(0,5)$ dans les premiers stades de vie. Mais ce rapport des sexes peut varier ensuite en fonction de la stratégie d'histoire de vie des individus qui est sexe dépendant (Cucherousset et al., 2005). Les migrations de reproduction pour l'accès aux zones de frayères ont lieu généralement de fin septembre à fin janvier (Ovidio et al., 1998). Ces migrations se font sur une plage de température allant de 6 à $12{ }^{\circ} \mathrm{C}$ et dépendent des fluctuations de débits entre plusieurs jours successifs (Baglinière et al., 1987). Les variables environnementales déclenchent les migrations de reproduction par vagues successives. Les gammes de distances parcourues sont très étendues (entre quelques dizaines de mètres à plusieurs dizaines de kilomètres). Le phénomène de homing n'est pas systématique (50 à $60 \%$; Baglinière, com. pers.). En 
revanche, un homing post reproducteur à savoir un retour des géniteurs vers l'habitat utilisé avant la migration est régulièrement observé (Ovidio, 1999). À noter que sur les rivières du Grand Ouest, une ségrégation entre les juvéniles (dans les affluents) et les adultes (dans les rivières) est observée. La croissance varie en fonction de la température (Baglinière \& Maisse, 1990) et de la disponibilité trophique (nombre et type de proies disponibles).

Les experts ont convergé pour retenir 5 stades de développement: œufs pondus, alevins vésiculés, alevins $(0+)$, juvéniles (1+) et adultes (>1+).

\subsubsection{Identification et hiérarchisation des facteurs influant les stades de développement}

Les étapes « identification et hiérarchisation des facteurs » menées successivement lors du séminaire sont restituées ici conjointement dans un souci de synthèse. Les schémas élaborés lors du séminaire ont été résumés en un seul tableau permettant d'avoir une vision synthétique des principaux facteurs influents (Tab. II). La liste des facteurs inventoriés n'est volontairement pas exhaustive afin de mettre en exergue les paramètres clé. Pour chaque stade de développement identifié précédemment, a été établi :

- un ordre décroissant de sensibilité aux différents processus mis en œuvre,

- des critères d'évaluation de ces processus,

- les investigations à mettre en œuvre pour être à même de renseigner ces critères.
L'échelle spatiale à laquelle s'opèrent les processus est également renseignée.

II est à noter que pour l'ensemble des stades de développement, le paramètre habitat a été particulièrement bien caractérisé par de nombreux auteurs. Les travaux de Haury et al. (1999) font une synthèse des principales caractéristiques décrites dans la littérature pour les différents stades de développement. Les courbes de préferenda élaborées dans le cadre de la méthode des micro-habitats décrivent quantitativement les besoins en termes de hauteur, vitesse et substrat auxquels il sera fait référence (Sabaton \& Gras, 1985 ; Souchon et al., 1989). Des courbes plus adaptées aux individus de grande taille existent aussi dans la littérature (Hayes, 1991 ; Louhi et al., 2008).

Pour chaque stade de développement, les paramètres sont présentés par ordre décroissant d'importance.

\subsubsection{CEufs pondus}

1/ Les surfaces de graviers-petits galets

La nature et la quantité de substrat sont estimées comme les paramètres les plus influents sur le nombre d'œufs pondus et sur la survie de ce stade de développement en résultant.

o À l'échelle du micro-habitat, le substrat de graviers-petits galets doit répondre aux critères suivants : un diamètre de granulométrie (entre 1 et $10 \mathrm{~cm}$ ), une épaisseur de graviers-petits galets entre 15 et $35 \mathrm{~cm}$ et une mobilité du substrat adaptée à la fois à la capacité de « creusement » qui dépend de la taille de la femelle (Hartman, 1969) tout en 


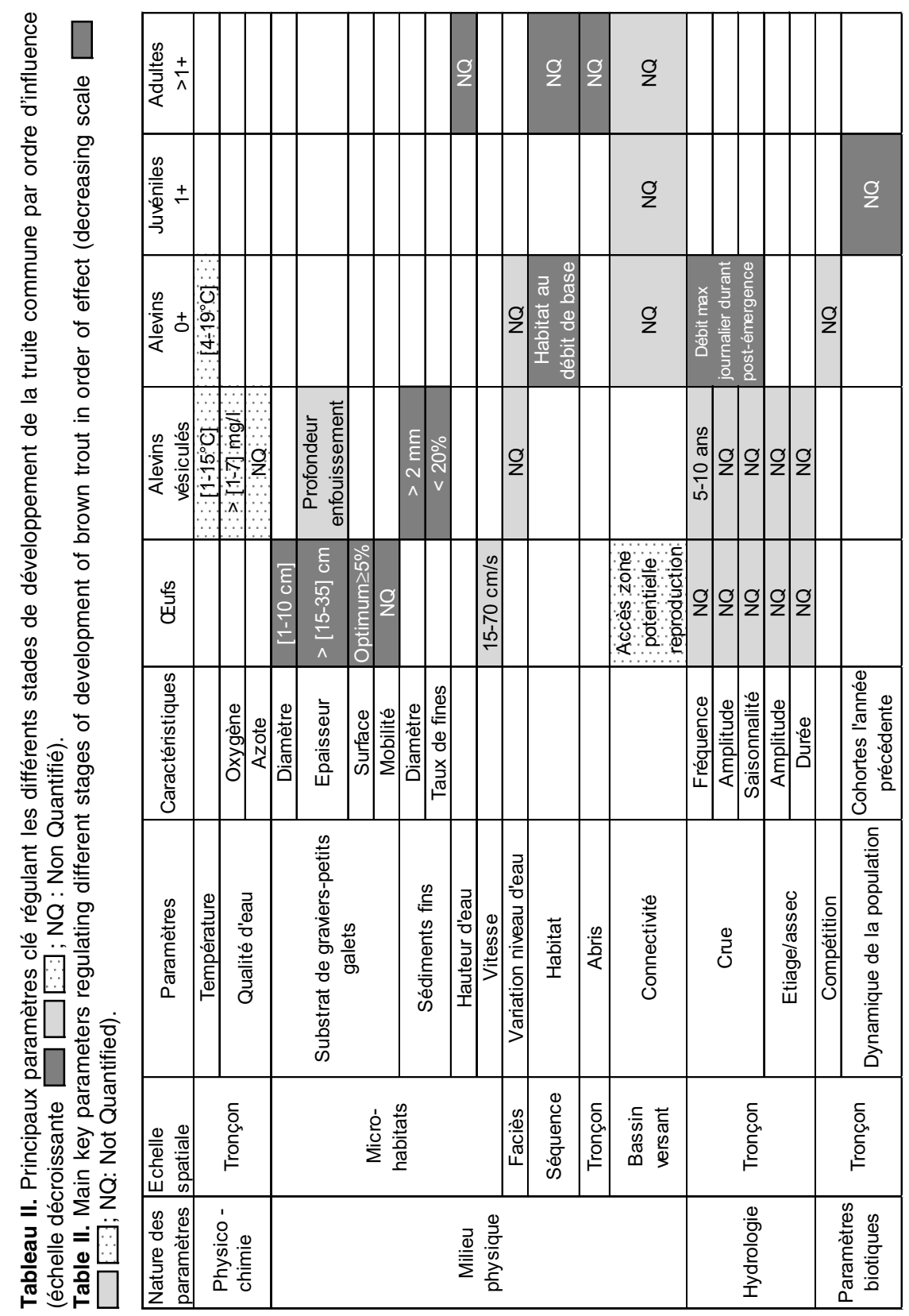


assurant une certaine cohésion pour limiter l'instabilité du substrat. Celleci dépend essentiellement de l'hétérogénéité des granulats et donc des modifications souvent anthropiques (comme l'érection de barrage).

o À l'échelle du tronçon, la surface de graviers-petits galets disponible est critique pour la survie de ce stade. Elle conditionne les mortalités par surcreusement des nids au cours de la saison de reproduction. En deçà de $1 \%$ de la surface mouillée, la surface de graviers-petits galets disponible limite la survie des œufs pondus. Elle est satisfaisante dès $3 \%$ et optimum au-delà de $5 \%$ (Delacoste, 1995).

\section{2/ Les vitesses}

À l'échelle du microhabitat, les conditions de vitesse sur les frayères constituent le second paramètre le plus influent sur ce stade de développement. Les vitesses du courant au fond de la colonne d'eau sur ces habitats de reproduction doivent respecter des plages de valeurs bien déterminées $\left(15-70 \mathrm{~cm} . \mathrm{s}^{-1}\right)$. Ces conditions de vitesses ont un poids plus ou moins important selon le type de cours d'eau.

La mise en perspective des conditions d'écoulement avec le régime hydrologique du site d'étude permet d'identifier l'influence du régime sur la dynamique des surfaces de reproduction. L'analyse des chroniques de débits (données historiques) permettra d'estimer la fréquence des assecs et des crues. Si des crues ou des assecs interviennent pendant la période de reproduction, il est important de déterminer leur intensité et leur durée dans le temps pour estimer leur niveau impact (arrêt de l'activité de ponte ou destructions des frayères).

3/ Connectivité vs. fragmentation du $\underline{\text { milieu }}$

À l'échelle du bassin versant, l'accès des géniteurs aux zones potentielles de reproduction (satisfaisant aux conditions de nature de substrat et de vitesse définies ci-dessus) est un élément prépondérant dans le succès reproducteur. Une recherche des zones potentielles de reproduction sur les affluents ou à l'amont permet d'évaluer si des surfaces favorables sont présentes à proximité du tronçon étudié. L'incidence de la connectivité est à estimer d'après l'évolution des conditions d'accessibilité des zones de reproduction en fonction des conditions hydrologiques, du nombre d'obstacles infranchissables et est à pondérer en fonction du déficit potentiel d'habitat de reproduction sur le cours d'eau principal.

Une comparaison des densités d'adultes avec la surface disponible permettra de mettre en regard les effectifs de géniteurs avec les potentialités du milieu.

Enfin, les facteurs anthropiques susceptibles d'influer sur ce stade sont les phénomènes d'érosion verticale pouvant conduire à l'incision du lit voire à la destruction du plancher alluvial, et de pavage (constitution, en fond de lit, d'une couche superficielle grossière peu ou pas mobilisable et empêchant la mobilisation de la sous-couche d'alluvions plus fines) ainsi que l'interruption du transport solide, totale ou partielle (tri granulométrique), induit par les aménagements (ouvrages transversaux, extractions, etc.). 
3.2.2.2 Alevins vésiculés (de l'éclosion à l'émergence)

Les paramètres déterminant la survie aux phases d'éclosion et d'émergence dans les nids sont la quantité de sédiments fins, les modifications importantes du débit et la qualité de l'eau.

\section{1/ Quantité de sédiments fins}

Un excès de sédiments fins $(<2 \mathrm{~mm})$ se traduit par un colmatage des frayères et un manque d'oxygénation des œufs. Le rôle des fractions granulométriques fines a été mis en évidence à différentes reprises puisqu'il a été montré que les mortalités dès le stade œuf sont plus précoces et plus accentuées sur les ruisseaux sur schiste comparés à ceux sur cornéenne (Massa et al., 2000). Ce dépôt de matières fines peut être estimé simplement par tamisage. Un taux de matières fines supérieur à $25 \%$ voire moins (20\%) correspond à une situation très critique pour ce stade (Liebig, 1998 ; Raleigh et al., 1986).

\section{2/ Profondeur d'enfouissement}

Elle n'a pas d'impact direct sur la survie à l'éclosion, mais par contre est susceptible d'influencer la dynamique d'émergence.

\section{3/ Variations des niveaux d'eau}

Les variations des niveaux d'eau induites par de forts étiages peuvent conduire à des exondations de nids qui occasionnent des mortalités sur les œufs. Des crues mobilisatrices entraînant ou non des sédiments fins peuvent enrayer le développement des œufs (mortalité par destruction des nids ou par hypoxie). En effet, le nombre de crues, leur intensité et leur localisation dans le temps restent des facteurs de pression importants pour la réussite de la ponte (Kondolf et al., 1991; Massa, 2000). Des crues importantes avec un débit mobilisateur du substrat ont une incidence sur la survie sous graviers par destruction des frayères (notamment des crues de fréquence de retour de 510 ans).

Enfin, les facteurs anthropiques susceptibles d'influer sur ce stade sont l'interruption du transport solide (ouvrages transversaux, extractions, etc.) et les variations des niveaux d'eau (tronçon court-circuité, éclusée, etc.) induites par les aménagements, ainsi que les dégradations de la qualité d'eau.

\section{3/ Qualité d'eau}

Des teneurs excessives en azote dans l'eau interstitielle peuvent provoquer des surmortalités (Massa, 2000). Les conditions de température et d'oxygène dissous sont des paramètres déterminants. Une analyse des chroniques de températures permet d'évaluer si les valeurs restent comprises entre $1,5^{\circ} \mathrm{C}$ et $15^{\circ} \mathrm{C}$ (Humpesch, 1985 ; Raleigh et al., 1986). Le taux minimal d'oxygène dissous évolue entre 1 à $7 \mathrm{mg} / \mathrm{L}$ entre le stade œuf et l'éclosion. Néanmoins, le taux critique de concentration en oxygène ne dépend pas uniquement de la quantité dissoute dans l'eau mais également de la vitesse de circulation de l'eau, du diamètre de l'œuf et du coefficient de diffusion dans sa membrane (Rombough, 1988).

Enfin, les facteurs anthropiques susceptibles d'influer sur ce stade sont les mêmes que ceux évoqués pour le facteur « variations des niveaux d'eau ». 


\subsubsection{Alevins $(0+)$}

Les paramètres les plus influents sur la survie des alevins sont les pointes de débit pendant la période de post-émergence et les conditions d'habitat (disponibilité et fonctionnalité : effets du colmatage) précédant les épisodes de crue. Viennent ensuite la connectivité et la température.

1/ Débit en phase de post-émergence et habitat en période de débit de base

Les fortes crues durant la période de post-émergence induisent des mortalités élevées sur les alevins. Ce phénomène a été observé sur de nombreux sites (Capra et al., 2003 ; Cattanéo, 2002 ; 2005 ; Cattanéo et al., 2002 ; Fahrner, 2010 ; Gouraud et al., 2001, $2008,2004)$. En revanche, les valeurs seuils des débits pénalisants n'ont pas pu être repérées par rapport à des descripteurs classiques en hydrologie. Par ailleurs, l'habitat en période de débit de base (débit avant l'arrivée de la crue) ainsi que l'amplitude entre le débit de pointe et le débit de base sont des paramètres qui modulent l'intensité de l'effet des crues sur ce stade de développement (Lauters, 1995 ; Valentin, 1995). Ces phénomènes ont été observés à l'échelle de séquences de faciès et sont synchrones à l'échelle des tronçons voire à l'échelle régionale (Fahrner et al., 2009). L'analyse des chroniques hydrologiques permet de décrire ces variables et de connaître les valeurs de débits prévalant durant la phase de post-émergence avec leurs caractéristiques (intensité, durée, saisonnalité). L'habitat au débit de base peut être évalué à l'aide d'un modèle d'habitat.

\section{2/ Connectivité}

À l'échelle du bassin versant, le développement du stade 0+ peut être modifié par une fragmentation du milieu réduisant la dispersion des individus, notamment en cas de capacité d'accueil limitante et en raison du caractère territorial de la truite. De fait, cette fragmentation peut renforcer les phénomènes de compétition intraspécifique (intra et inter classe d'âge) et de densité dépendance (mortalité et croissance). Elle peut alors influer sur les choix d'habitat (Heggenes et al., 1999) et de stratégie de vie de l'individu (Acolas et al., 2012). À l'échelle du tronçon, l'instabilité du milieu ainsi que les fluctuations de niveaux d'eau peuvent également induire une mauvaise répartition des individus par rapport aux habitats favorables. L'effet négatif des variations de débit a été démontré notamment lorsque ces dernières se produisent au moment de l'émergence et de nuit car les alevins choisissent alors des habitats où la profondeur d'eau est extrêmement faible (Bardonnet et al., 2006 ; Roussel et Bardonnet, 1999).

La connectivité du système est à évaluer en focalisant sur l'aval du tronçon d'étude et en estimant la qualité de l'obstacle à franchir en période de dévalaison (risque de mortalité : turbines, chute d'eau) pour rejoindre des zones plus adaptées à la taille des $0+$. L'estimation de la disponibilité et de l'état du linéaire à l'aval (sur le cours d'eau principal ainsi que les affluents) est à effectuer jusqu'au premier obstacle pénalisant pour la migration vers l'aval.

\section{3/ Température}

La température a un effet direct sur la survie et la taille de l'alevin à l'éclosion 
et sur la croissance du juvénile lors de sa première saison de croissance (Baglinière \& Maisse, 1990). Les travaux de Spaas (1958), Bishai (1960), Frost \& Brown (1967), Gardside (1973), Elliott (1981) ont permis de distinguer 3 plages de température : une plage optimale $\left(4-19^{\circ} \mathrm{C}\right)$, une plage critique pour les faibles températures $\left(0-4{ }^{\circ} \mathrm{C}\right)$ et une plage critique pour les fortes températures $\left(19-30^{\circ} \mathrm{C}\right)$. Le seuil de $7^{\circ} \mathrm{C}$ en dessous duquel la croissance n'est pas effective est, cependant, à retenir pour le genre Salmo à la différence du genre Salvelinus (Baglinière, com. pers.). Les courbes de preferenda thermiques établies par Bovee (1982) à partir d'observations in situdonnent des valeurs critiques pour les fortes températures. Ces dernières sont similaires à celles obtenues à partir des expérimentations en milieu contrôlé (Elliott, 1981). Les effets de ce paramètre à l'échelle du tronçon ont été observés sur des populations de truite de cours d'eau bas-normand (Gouraud et al., 2001).

L'influence de la température doit être analysée en confrontant les densités d'alevins aux températures maximales observées. Ces dernières doivent être mises en perspectives par rapport aux valeurs seuils données dans la littérature.

D'autres phénomènes tels que la prédation, les teneurs élevées en matières en suspension (transparence de l'eau), l'apparition de pathologies telles que la PKD (Proliferative Kydney Disease, maladie proliférative des reins) peuvent agir sur les abondances et la croissance des $0+$. Ces phénomènes n'ont, cependant, pas été jugés prépondérants.

\subsubsection{Juvéniles (1+)}

Les paramètres prépondérants retenus sont l'abondance de la cohorte l'année précédente ainsi que la connectivité du milieu.

\section{1/ Densité de 0+ l'année précédente}

Sur les tronçons regroupant les zones de reproduction et de croissance, les densités de 1+ sont fortement dépendantes des densités de 0+ présentes l'année précédente. Pour les cours d'eau où les zones de reproduction et de croissance sont disjointes et/ou la croissance est élevée (cours d'eau normands et bretons notamment), la densité entre les deux classes d'âge reste corrélée (Baglinière \& Champigneulle, 1982). Néanmoins, cette relation peut apparaître moins évidente pour deux raisons: une migration à l'intérieur du système rivière voire en mer ou une rentrée des juvéniles 1+ au cours de leur deuxième année de vie dans la pêcherie sportive en raison de leur taux de croissance.

\section{2/ Connectivité amont-aval}

La connectivité amont-aval joue un rôle important sur la dispersion et le niveau d'abondance en raison de phénomènes de déplacements / migrations chez les individus 1+. La connectivité du système est à évaluer à l'amont et à l'aval du tronçon d'étude en axant la priorité sur les zones aval. Les critères à évaluer sont i/ la possibilité de pouvoir rejoindre un affluent refuge en cas de crue importante, ii/ les caractéristiques de l'obstacle à franchir en dévalaison (risque de mortalité : turbines, chute d'eau) pour rejoindre des zones plus adaptées à la taille des 1+ (profondeur). 
La cartographie des obstacles à l'amont est à réaliser en distinguant la taille des ouvrages (prise d'eau ou grand réservoir). À l'aval, l'estimation du linéaire disponible et de son état (sur le cours d'eau principal ainsi que les affluents) est à effectuer jusqu'au premier obstacle pénalisant pour la dévalaison.

D'autres paramètres peuvent jouer sur l'abondance de cette cohorte à savoir l'habitat (disponibilité de zones peu profondes plus ou moins courantes possédant toutes sortes d'abris, les teneurs en matières en suspension, la qualité d'eau, les conditions de températures, l'apparition de pathogènes (PKD), le déversement d'individus issus de pisciculture). Ces paramètres n'ont cependant pas été jugés prépondérants.

\subsubsection{Adultes $(>1+)$}

Pour les adultes, le respect de conditions d'habitat satisfaisantes, la présence d'abris ainsi que la connectivité du milieu déterminent principalement l'abondance de ce stade.

\section{1/ Profondeur, abris, habitat}

L'habitat des adultes est à évaluer principalement au débit en période d'étiage. L'habitat recouvre les abris, la surface pondérée utile (SPU) avec une courbe de preferenda la plus adaptée et la température moyenne.

\section{2/ Connectivité}

La connectivité amont-aval est à évaluer en prenant en considération la nécessité pour les adultes de rejoindre des sites de reproduction parfois très éloignés des sites de croissance et fréquemment situés en tête de bassin. Dans le sens de la montaison, la truite commune adulte possède toutefois des capacités de franchissement d'obstacles plus importantes que les espèces holobiotiques de nos cours d'eau (Ovidio et al., 2007). Dans le sens de la dévalaison, le homing post-reproducteur fréquemment observé chez les géniteurs qui survivent implique le passage des ouvrages dans le sens de la dévalaison avec toutes les conséquences possibles (blessure, mortalité) dans le cas d'ouvrages hydroélectriques.

D'autres paramètres défavorables liés à l'impact des activités humaines peuvent jouer sur la présence et l'abondance des adultes (teneurs élevées en matières en suspension, températures supérieures à $20^{\circ} \mathrm{C}$, mauvaise qualité d'eau, pression de pêche importante).

\subsubsection{Sur l'ensemble des stades de développement}

Un consensus a émergé sur la difficulté d'identifier des critères robustes permettant de statuer sur le fonctionnement des populations de truite en termes d'abondance, de biomasse, de structure de population et d'utilisation de l'habitat (taux d'occupation). Il en est de même pour la viabilité de la population à savoir le nombre minimal d'individus nécessaires pour assurer la survie d'une population. Cependant, indépendamment de la stratégie démographique ou de vie adoptée par la population de truite ou ses individus (migration vs. sédentarisation), une densité de juvéniles $(0+)$ entre 30 et $50 \mathrm{ind} / 100 \mathrm{~m}^{2}$ sur les zones apicales des têtes de bassin a été proposée comme un critère de bon fonctionnement ou tout au moins de bonne santé de la population (Baglinière, com. pers.). 
Des critères sur la distribution des abondances par effectif de taille seraient également nécessaires à déterminer pour donner une image fonctionnelle de la population.

Quoi qu'il en soit, l'élaboration de critères biologiques nécessiterait de connaître la très grande variabilité des structures de populations observées en fonction de la localisation géographique. Si on pose l'hypothèse que la comparaison à des conditions de référence permet de mesurer un impact, cette comparaison demande de connaître l'enveloppe des variabilités des paramètres biologiques observés dans des situations où l'occupation humaine n'a pas modifié significativement les processus physiques, biogéochimiques et écologiques. Cette connaissance n'a pas été jugée ici suffisante pour permettre de définir des critères biologiques génériques susceptibles d'évaluer le fonctionnement d'une population de truite à une échelle locale. Dans le cas de la mesure de l'impact d'un usage de l'écosystème à l'échelle locale, la difficulté d'avoir à disposition un site de référence comparable au site impacté a été souligné.

Enfin, il ressort quel que soit le type de milieu, que les stades considérés comme les plus vulnérables sont les jeunes stades. Les individus adultes $(>1+)$ sont surtout susceptibles d'être limités par l'habitat lorsque le recrutement est important.

Sur un même site, les différences inter-annuelles de densité sont peu reliées à l'habitat mais ce lien est cependant observé en cas de changement de qualité de substrat et de fort recrutement annuel en $0+$ (capacité d'accueil atteinte). En revanche, les abondances inter-sites sont liées à l'habitat et à la position de la station d'étude sur le réseau (leur accès dépendant de l'hydrologie et de la position). Pour un milieu donné, de grandes différences de surface d'habitat sont nécessaires pour observer une réponse sur la population dans la mesure où cette réponse est plus reliée au taux d'hétérogénéité spatial qu'à la surface totale d'habitat.

En conclusion, les connaissances actuelles permettent d'apprécier le fonctionnement des populations de truite via la sélection de critères sur le milieu physique. Cette sélection doit se baser sur les déterminants physiques qui expliquent la dynamique et le fonctionnement de la population. Même si des seuils ne peuvent être définis pour l'ensemble des paramètres clé du milieu qui ont été identifiés, des connaissances sont disponibles sur la manière dont certains paramètres influent sur les populations.

\section{DISCUSSION}

\subsection{Limites méthodologiques}

Le type de méthode retenue ici (recueil d'avis d'experts via une réflexion collective) est largement utilisé en écologie (méthode delphi (Rowe \& Wright, 1999)). Ce type de démarche est particulièrement bien adapté au caractère heuristique de l'écologie. En effet, comme le souligne Lévêque (2001), la nature et la complexité des phénomènes en écologie, ne permet pas à l'instar de la physique ou de la génétique, de créer un édifice important de 
lois organisées. L'écologie a alors recours à la formulation d'hypothèses qu'on adopte provisoirement comme idées directrices dans la recherche des faits. Pour être appliquées, ces hypothèses doivent cependant faire l'objet de partage scientifique mais pas forcément de l'unanimité. De fait, la mise en oeuvre de ce type d'exercice permet de mettre en exergue les convergences et les divergences scientifiques. La mise en évidence des controverses/doutes scientifiques permet de faire ressortir le véritable niveau de connaissances acquis et sur quelle échelle spatio-temporelle les connaissances sont à préciser et/ou à acquérir avec leur niveau d'incertitude.

Les limites de l'exercice viennent indubitablement du fait que les critères ont été définis par une poignée d'experts et ne constituent que le reflet des avis d'une partie de la communauté scientifique. En revanche, la démarche présente l'avantage de faire ressortir des multiples résultats scientifiques disponibles dans la littérature sur la biologie de la truite, un consensus sur les paramètres les plus influents et sur lesquels un effort particulier de caractérisation doit être mené lors d'un diagnostic du fonctionnement d'une population de truite. Pour compléter cette démarche, il pourrait alors être envisagé :

i) de soumettre les critères retenus à d'autres scientifiques français et étrangers (soit via une consultation par courrier, soit via un séminaire). Les experts sélectionnés ici étant spécialisés sur l'influence des paramètres physiques sur la biologie, la démarche serait à compléter avec des experts dans d'autres domaines (physiologie, génétique...). Les résultats pourraient également être confrontés aux visions des acteurs chargés de la mise en œuvre des diagnostics du fonctionnement des écosystèmes afin d'intégrer un éventail plus large de types de connaissances ;

ii) d'avoir une démarche expérimentale et de tester les critères retenus pour savoir s'ils permettent d'aboutir à l'objectif recherché, à savoir diagnostiquer l'état de santé des populations de truite et ainsi de l'écosystème aquatique en tête de bassins dont la truite est l'espèce représentante la plus significative en France.

\subsection{Nature des critères retenus}

II ressort du séminaire que, dans l'état actuel des connaissances, le diagnostic peut être dressé seulement en se basant sur les paramètres physiques cruciaux pour la biologie (et plus difficilement sur les paramètres biologiques). Pour l'ensemble des stades de développement, les paramètres identifiés sont l'habitat (avec une attention particulière sur le substrat, les conditions d'écoulement, la température et la qualité d'eau), l'hydrologie et la connectivité.

Cependant, dans un premier temps, réaliser le même exercice mais en se basant sur l'analyse partagée de suivis biologiques mis en œuvre sur des durées suffisantes ( $>5$ ans) et sur des milieux physiques variés permettrait probablement d'aboutir à l'émergence de critères quantitatifs sur ces paramètres biologiques. 
Dans un second temps, pour parfaire ce type de diagnostic, une identification des valeurs des paramètres du milieu se révélant pénalisantes pour les populations serait à réaliser via des analyses multi-sites. Si les tendances d'évolution de la population en lien avec ces paramètres sont connues à l'heure actuelle, des lois généralisées ou adaptées à un site restent à élaborer pour disposer des valeurs seuils, d'amplitude, de gradients sur les débits de crue, les débits mobilisateurs du substrat, les vitesses d'entraînement, les tolérances thermiques et le degré de fragmentation...

En mettant en regard les processus physiques reflétant divers degrés d'altération et la structure des populations, des connaissances émergeront sur le fonctionnement des populations. Cette connaissance est essentielle pour dépasser le simple diagnostic sur les paramètres physiques qui ne retranscrit pas la variabilité des réponses des populations en fonction du degré d'altération, l'importance du contexte physique et les phénomènes de compensation qui se mettent en place. L'acquisition de cette compréhension plus fine des mécanismes est nécessaire pour identifier les leviers d'actions de restauration. C'est dans ce sens que la DCE a mis la biologie au centre de la démarche. Afin de relever ce défi, il reste nécessaire d'établir à terme des critères biologiques sans se cantonner aux critères physiques cruciaux pour la biologie. Pour ce faire, des recherches doivent être mises en place pour appréhender la variabilité des paramètres biologiques, les échelles spatio-temporelles mises en jeu et les processus fonctionnels.
4.3 Variabilités biologiques à différentes échelles spatio-temporelles

L'enveloppe de variabilité des réponses locales aux paramètres du milieu doit être caractérisée. Dans ce sens, les travaux récents de Fahrner (2010) apportent des éléments donnant une vision d'ensemble des réponses des populations de truite aux déterminants physiques selon le type de milieu où l'on se trouve. Une analyse multisites des chroniques pisciaires allant de 4 à 10 ans sur 53 sites répartis dans le Massif Central, les Alpes et les Pyrénées ont cherché, notamment, à mettre en évidence les paramètres clé du milieu pour la structuration des populations, en comparant les réponses observées en milieu naturel à celles constatées en tronçon à débit régulé. Les résultats montrent clairement que les densités de truite sont négativement corrélées à la largeur moyenne de la station, et ce quel que soit le stade, le massif et l'influence exercée sur le tronçon (tronçon naturel ou régulé). Le rôle de l'hydrologie est plus marqué en tronçon court-circuité.

Des approches doivent également être développées pour appréhender les échelles spatio-temporelles auxquelles les différents processus interviennent. Pour ce faire, des recherches doivent être menées à des échelles spatiales et temporelles élargies, pour acquérir des connaissances sur la dynamique des communautés biologiques à l'échelle d'un bassin et les interactions entre les processus locaux et ceux agissant à plus large échelle. De plus, les liens avec les autres compartiments de l'écosystème aquatique doiventêtre identifiés. 
II importe alors de développer des travaux pour :

- mettre en relation les caractéristiques du bassin versant (hydrologie, sol...), les modes d'évolution de la morphologie des masses d'eau (type géomorphologique, géométrie hydraulique, connexion des annexes hydrauliques) avec la diversité biologique animale et végétale associée (structures et fonctions) ;

- mettre en évidence les influences des ruptures de connectivité (notion d'impacts cumulés) sur la disponibilité en habitats fonctionnels (alimentation, refuge, reproduction) et la viabilité des communautés locales ;

- conduire une évaluation globale du fonctionnement à l'échelle du bassin.

Des analyses systémiques du bassin seraient à réaliser pour caractériser le fonctionnement écologique en lien avec l'hydrologie, l'hydromorphologie, la thermie et la physico-chimie.

\subsection{Processus fonctionnels}

Les activités agricoles, l'urbanisation, les aménagements du paysage, des cours d'eau et de leur espace de fonctionnement (lit majeur, espace de mobilité), les pollutions diverses, les introductions d'espèces, ainsi que l'exploitation des ressources vivantes sont devenus des facteurs de forçage majeurs de la qualité et de la fonctionnalité écologique des milieux aquatiques. Ces contraintes sollicitent les capacités de résistance, de plasticité et d'adaptation des organismes, ce qui se traduit par une influence globale sur la résistance des écosystèmes aux perturbations et sur leurs possibilités de résilience. À ces modifications directement liées aux activités humaines à l'échelle locale s'ajoutent celles provenant des changements globaux, notamment climatiques (modifications du régime thermique et de la pluviométrie avec leurs conséquences sur la répartition et les déplacements des espèces). Cette dynamique et cette résilience sont liées à 3 grandes caractéristiques de processus: physique (morphodynamique fluviale), biogéochimique (flux de matières organiques et énergie métabolique), écologique (possibilité de dispersion et de recolonisation des espèces). Dans un écosystème qui «fonctionne bien », les processus fonctionnels maintiennent une diversité physique et biologique, assurant la réversibilité du système face aux perturbations d'origine anthropique ou naturelle.

Si les processus permettant le maintien d'une diversité physique sont bien décrits et facilement appréhendables, il n'en va pas de même des processus permettant le maintien de la diversité biologique. Les métriques biologiques permettant d'évaluer si la population a la capacité de faire face aux perturbations d'origine anthropique ou naturelle ne sont pas connues. Par ailleurs, la comparaison à une référence s'avère souvent peu satisfaisante (pas de site disponible comparable la plupart du temps) et l'écart en biomasse ou en densité sur un site éloigné de ce qu'on trouve sur un site de référence ne traduit pas de façon fiable un degré de perturbation.

Aussi, sur les têtes de bassin où la diversité pisciaire est faible, le rôle fonctionnel de ces processus doit être appréhendé en intégrant i) le niveau d'organisation biologique individuel 
(trajectoire et trait d'histoire de vie) au sein d'une population ou d'une métapopulation (trait populationnel, démographie, taille efficace et diversité génétique) et ii) la composante individuelle dans le fonctionnement global de l'écosystème (flux d'individus et d'énergie, communautés et réseaux trophiques).

4.5 Frontière entre question scientifique et choix sociétal

En faisant du «bon état » des masses d'eau l'objectif même des politiques de gestion des ressources hydriques, la DCE a rendu nécessaire un double débat à la fois politique (comment définir ce qui est bon ?) et technique (comment caractériser le bon état?). Sur le plan politique, ces notions théoriques de bon état ou bon potentiel sont débattues et définies localement par les différents acteurs pour chaque masse d'eau. Des connaissances scientifiques doivent être apportées pour nourrir ces débats et servir de point d'appui à ces échanges. Cet objectif ambitieux pose à la communauté scientifique bien des questions conceptuelles : qu'est-ce qu'un écosystème qui fonctionne bien ? et méthodologiques : quels sont les descripteurs pertinents pour juger du bon fonctionnement et comment les mesurer? C'est sur ces questions conceptuelles et méthodologiques que l'approche développée ici avait pour vocation de répondre.

La frontière entre question scientifique et question de l'usage des écosystèmes relevant d'un choix sociétal n'est, cependant, pas si nette qu'il y paraît. Certains experts ont remis en question, lors du séminaire, la notion de bon fonctionnement sur le plan scientifique. Ce bon fonctionnement est perçu par certains experts comme relevant plus de la sphère politique dans le sens qu'il fait appel à un choix de société. Le bon fonctionnement et/ou le bon état de santé pourrait être défini comme la caractéristique d'une population qui au travers de la perception qu'en a la société procure un sentiment de satisfaction à cette même société (besoins/ usages, ressources, valeurs/patrimoine, etc.) ou de service rendu. Le bon fonctionnement d'un écosystème notamment aquatique compte tenu de l'empreinte humaine grandissante sur les milieux correspond typiquement à un service écosystémique.

Les questions relevant du champ scientifique concernent l'évaluation des processus mis en œuvre, l'identification des perturbations avec la mise en évidence de l'origine anthropique et de l'origine naturelle. Cependant, le fait que des états de référence n'existent que rarement à une échelle locale oblige le scientifique à se prononcer dans la définition $d u$ «bon ». De manière plus générale, le choix des outils retenus pour l'évaluation du fonctionnement n'est pas anodin. Ce choix participe de manière indirecte à la définition du «bon fonctionnement ». Dans ce sens, des auteurs dans la littérature remettent en cause la neutralité des outils. Lascoumes \& Le Gales (2005) soulignent que les outils sont supposés être construits et définis d'après la fonction qu'ils doivent remplir. Cette fonction est de fournir une base sur laquelle construire l'action publique. II faut rompre avec cette illusion de la neutralité des instruments et reconnaître, en revanche, leur force 
d'action en raison de la connaissance qu'ils apportent et du cadre normatif qu'ils proposent. Une partie de la décision se prend dans le débat technique. Le développement d'outils par un petit groupe d'acteurs peut avoir un impact phénoménal, avec parfois des effets propres aux outils qui vont au-delà des souhaits des développeurs.

Aussi, le partage des connaissances et l'émergence de consensus scientifiques sont essentiels pour que le choix des outils retenus soit le plus rationnel possible et le mieux adapté à l'évaluation du fonctionnement des écosystèmes. Dans ce sens, il est important de mettre en œuvre des démarches susceptibles de faire émerger ces consensus. La création d'une instance de partage des résultats acquis via des suivis d'écosystèmes sur du long terme et sur différents sites permettrait probablement de faire émerger les critères biologiques qui nous manquent à l'heure actuelle.

Enfin, cette notion de fonctionnement des écosystèmes avancera dans le domaine scientifique si les objectifs politiques restent bien distincts des objectifs scientifiques même s'il existe un lien entre les deux qui sont les enjeux sociétaux. L'élaboration des paradigmes est un processus long qui ne peut être tournée uniquement vers la satisfaction des besoins à court terme, au risque de faire de l'écologie une science instrumentalisée (Lévêque, 2001).

\section{RÉFÉRENCES}

Acolas M.L., Labonne J., Baglinière J.L. \& Roussel J.M., 2012. The role of body size versus growth on the decision to migrate: a case study with Salmo trutta. Naturwissenschaften 99 : 11-21.

Amoros C. \& Petts G.E., 1993. Hydrosystèmes fluviaux. Collection Écologie $\mathrm{n}^{\circ}$ 24. Masson, Paris, $300 \mathrm{p}$.

Baglinière J.L., 1999. Introduction: The brown trout (Salmo trutta L.): its origin, distribution and economic and scientific significance. In : Baglinière J.L. \& Maisse G. (Eds.), Biology and Ecology of the Brown and Sea trout, SpringerPraxis, Chichester, UK, 1-12.

Baglinière J.L. \& Champigneulle A., 1982. Densité des populations de Truite commune (Salmo trutta L.) et de juvéniles de Saumon atlantique (Salmo salar L.) sur le cours principal du Scorff (Bretagne) : preferendums physiques et variations annuelles (1976-1980). Acta Oecologica Oecologia Applicata 3 : 241-256.

Baglinière J.L. \& Maisse G., 1990. La croissance de la truite commune (Salmo trutta L.) sur le bassin du Scorff. Bull. Fr. Pêche Piscic. 318 : 89-101.

Baglinière J.L. \& Maisse G., 1991. Biologie de la truite commune (Salmo trutta L.) dans les rivières françaises. In : Baglinière J.L. \& G. Maisse (Eds.), La truite - Biologie et écologie, INRA Editions, 25-46.

Baglinière J.L., Maisse G., Lebail P.Y. \& Prévost E., 1987. Dynamique de la population de truite commune (Salmo trutta L.) d'un ruisseau breton (France) : les géniteurs migrants. Acta Oecologica Oecologica Applicata 8(3) : 201-215.

Bardonnet A., Poncin P. \& Roussel J.M., 2006. Brown trout fry move inshore at night: a choice of water depth or velocity? Ecology of Freshwater Fish 15(3) : 309-314.

Bishai H.M., 1960. Upper lethal temperatures for larval salmonids. ICES. Journal of Marine Science 25 : 129-133.

Botosaneanu L., 1988. Zonation et classification biologique des cours d'eau : développements récents, alternatives, 
perspectives. Atti XV Congr. naz. ital. Ent. 33-61.

Bovee K.D., 1982. A guide to stream habitat analysis using the Instream Flow Incremental Methodology. Fish and Wildlife Service, Office of Biological Services. Fort Collins, Colorado, $248 \mathrm{p}$.

Bravard J.P. \& Petit F., 1997. Les cours d'eau : dynamique du système fluvial. Collection U, Armand Colin, Paris, $222 \mathrm{p}$.

Capra H., Sabaton C., Gouraud V., Souchon Y. \& Lim P., 2003. A population dynamics model and habitat simulation as a tool to predict brown trout demography in natural and bypassed stream reaches. River Research and Applications 19(5-6) : 551-568.

Cattanéo F., 2002. Influence de l'hydrologie sur les populations et assemblages piscicoles : mythes et réalités. Thèse de doctorat, $320 \mathrm{p}$.

Cattanéo F., 2005. Does hydrology constrain the structure of fish assemblages in French streams? Local scale analysis. Arch. Hydrobiol. 164(3) : 345365.

Cattanéo F., Lamouroux N., Breil P. \& Capra $H ., 2002$. The influence of hydrological and biotic processes on brown trout (Salmo trutta) population dynamics. Canadian Journal of Fisheries and Aquatic Sciences 59 : 12-22.

Cucherousset J., Ombredane D., Charles K., Marchand F. \& Baglinière J.L., 2005. A continuum of life history tactics in a brown trout (Salmo trutta) population. Canadian Journal of Fisheries and Aquatic Science 62 : 1600-1610.

Delacoste M., 1995. Analyse de la variabilité spatiale de la reproduction de la truite commune (Salmo trutta L.) - Étude à l'échelle du micro et du macrohabitat dans 6 rivières des Pyrénées centrales. Thèse de doctorat, $193 \mathrm{p}$.
Elliott J.M., 1981. Some aspects of thermal stress on freshwater teleosts. In: Pickering A.D. (Ed.), Stress and fish 209-245.

Fahrner G., 2010. Typologie des impacts potentiels des ouvrages hydroélectriques sur les populations de truite situées en aval. Doctorat ParisTech Sciences de l'environnement, $161 \mathrm{p}$.

Fahrner G., Villeneuve B., Gouraud V., Capra H. \& Souchon Y., 2009. Spatial scale and degree of synchrony in brown trout (Salmo trutta) population dynamics. Proceedings of the 7th International Conference on Ecohydraulics, Concepcion, Chili.

Frissell C.A., Liss W.J., Warren C.E. \& Hurley M.D., 1986. A hierarchical framework for stream habitat classification: viewing streams in a watershed context. Environmental Management 10(2) : 199-214.

Frost W.E. \& Brown M.E., 1967. The trout. Collins, St. James' Place, London, 286 p.

Garside, E.T., 1973. Ultimate upper lethal temperature of Atlantic salmon (Salmo salar). Canadian Journal of Zoology $51: 898-900$.

Gouraud V., 1999. Étude de la dynamique de populations de truite commune (Salmo trutta L.) à l'aide d'un modèle déterministe. Application sur un bassin bas-normand et sur un bassin pyrénéen. Doctorat ENGREF - Sciences de l'eau, $182 \mathrm{p}$.

Gouraud V., BaglinièreJ.L., Baran P., Sabaton C., Lim P. \& Ombredane D., 2001. Factors regulating brown trout populations in two french rivers: Application of a dynamic population model. Regulated Rivers: Research and Management 17 : 557-569.

Gouraud V., Sabaton C. \& Capra H., 2004. Role of habitat variability in trout population dynamics: Application of a dynamic population model to three French rivers. Hydroécologie Appliquée 14(1) : 221-244. 
Gouraud V., Capra H., Sabaton C., Tissot L., Lim P., Vandewalle F., Fahrner G. \& Souchon Y., 2008. Long-term simulations of the dynamics of trout populations on river reaches bypassed by hydroelectric installations - Analysis of the impact of different hydrological scenarios. River Research and Applications 24 : 11851205.

Hartman G.F., 1969. Reproductive biology of the gerrard stock rainbow trout. In: Northcote T.G. (Ed.), Symposium on salmon and trout in streams. University of British Columbia, Vancouver, B.C., 5367.

Haury J., Ombredane D. \& Baglinière J.L., 1999. The habitat of the brown trout (Salmo trutta L.) in water courses. Biology and ecology of the brown and sea trout. Spinger-Praxis ed., 37-89.

Haury J., Peltre M., Trémolières M., Barbe J., Thiébaut G., Bernez I., Daniel H., Chatenet P., Haan-Archipof G., Muller S., Dutartre A., Laplace-Treyture C., Cazaubon A. \& Lambert-Servien E., 2006. A new method to assess water trophy and organic pollution - the Macrophyte Biological Index for Rivers (IBMR): its application to different types of river and pollution. Hydrobiologia 570(1) : 153-158.

Hayes J., 1991. Microhabitat used by large brown trout for drift feeding. Freshwater Catch 45 : 7-9.

Heggenes J., Baglinière J.L. \& Cunjak R.A., 1999. Spatial niche variability for young Atlantic salmon (Salmo salar) and brown trout ( $S$. trutta) in heterogeneous streams. Ecology of Freshwater Fish 8 (1) : 1-21.

Hobbs D.F., 1940. cited by Allen, 1951, The Horokiwi stream: a study of a trout population. New Zealand Marine Department Fisheries Bulletin 10 : 1-238.

Huet M., 1949. Aperçu des relations entre la pente et les populations piscicoles des eaux courantes. Schweiz. Z. Hydrol. 11(3-4) : 332-351.

Humpesch U.H., 1985. Inter- and intra- variation in hatching success and embryonic development of five species of salmonids and Thymallus thymallus. Arch. Hydrobiol. 104(1) : 129-144.

Illies J. \& Botosaneanu L., 1963. Problèmes et méthodes de la classification et de la zonation écologique des eaux courantes, considérées surtout du point de vue faunistique. Mitteilung internationale Vereinigung Limnologie 12 : 1-57.

Kondolf G.M., Cada G.F., Sale M.J. \& Felando T., 1991. Distribution and stability of potential salmonid spawning gravels in steep boulder-bed streams of the eastern Sierra Nevada. Trans. Amer. Fish. Soc. 120 : 177-186.

Kondolf G.M., Montgomery D.R., Piègay H. \& Schmitt L., 2003. Geomorphic classification of rivers and streams. In Tools in fluvial geomorphology. John Wiley \& Sons ed., 169-202.

Lascoumes P. \& Le Gales P., 2005. Gouverner par les instruments. Pôle Sud, $N^{\circ} 23$ - 2005, 200-202.

Lauters F., 1995. Impact sur l'écosystème aquatique de la gestion par éclusées des ouvrages hydroélectriques. Thèse de doctorat, $224 \mathrm{p}$.

Lévêque C., 2001. Écologie - De l'écosystème à la biosphère. Dunod. Paris, 502 p.

Liebig H., 1998. Étude du recrutement de la truite commune (Salmo trutta L.) d'une rivière de moyenne montagne. Thèse de doctorat, $201 \mathrm{p}$.

Louhi P., Mäki-Petäys A. \& Erkinaro J., 2008. Spawning habitat of Atlantic salmon and brown trout: general criteria and intragravel factors. River Res. Applic. 24 : 330-339.

Maisse G. \& Baglinière J.L., 1999. Biology of the brown trout (Salmo trutta L.) in french rivers. In: Baglinière J.L. \& 
Maisse G. (Ed.), Biology and Ecology of the Brown and Sea trout 15-35.

Malavoi J.R., 1989. Typologie des faciès d'écoulement ou unités morphodynamiques des cours d'eau à haute énergie. Bull. Fr. Pêche Piscic. 315 : 189-210.

Massa F., 2000. Sédiments, physico-chimie du compartiment interstitiel et développement embryolarvaire de la truite commune (Salmo trutta) : étude en milieu naturel anthropisé et en conditions contrôlées. Thèse de doctorat, $178 \mathrm{p}$.

Massa F., Baglinière J.L., Prunet P. \& Grimaldi C., 2000. Survie embryo-larvaire de la truite (Salmo trutta) et conditions chimiques dans la frayère. Cybium 24(3) Suppl. 129-140.

Mondy C.P., Villeneuve B., Archaimbault V. \& Usseglio-Polateraa P., 2012. A new macroinvertebrate-based multimetric index (I2M2) to evaluate ecological quality of French wadeable streams fulfilling the WFD demands: A taxonomical and trait approach. Ecological Indicators 18 : 452-467.

Montgomery D., Beamer E.M., Pess G.R. \& Quinn T.P., 1999. Channel type and salmonid spawning distribution and abundance. Canadian Journal of Fisheries and Aquatic Sciences 56(3) : 377-387.

Ovidio M., 1999. Cycle annuel d'activité de la truite commune (Salmo trutta L.) adulte : étude par radio-pistage dans un cours d'eau de l'ardenne belge. Bull. Fr. Pêche Piscic. 352 : 1-18.

Ovidio M., Baras E., Goffaux D., Birtles C. \& Philippart J.C., 1998. Environmental unpredictability rules the autumn migrations of trout (Salmo trutta) in the Belgian Ardennes. Hydrobiologia 372 : 262-273.

Ovidio M., Capra H. \& Philippart J.C., 2007. Field protocol for assessing small obstacles to migration of brown trout Salmo trutta, and European grayling Thymallus thymallus: a contribution to the management of free movement in rivers. Fisheries Manag. Ecol. 14(1) : 41-50.

Pont D., 2009. Impacts potentiels du changement climatique sur les communautés et les populations piscicoles : Bilan des programmes GICC. Séminaire Onema programme GICC (MEEDDAT) Changement climatique, impacts sur les milieux aquatiques et conséquences pour la gestion, Paris, 29/06/2009.

Pont D., Hugueny B., Beier U., Goffaux D., Melcher A., Noble R., Rogers C., Roset N. \& Schmutz S., 2006. Assessing river biotic condition at a continental scale: a European approach using functional metrics and fish assemblages. J. Appl. Ecol. 43(1) : 70-80.

Raleigh R.F., Zuckermann L.D. \& Nelson P.C., 1986. Habitat suitability index models and instream flow suitability curves: brown trout. Fish and Wildlife Service, Office of Biological Service, $65 \mathrm{p}$.

Rapport D.J., Costanza R. \& McMichael A.J., 1998. Assessing ecosystem health. Trends in Ecology and Evolution 13(10) : 397-402.

Ricker W.E., 1934. An ecological classification of certain Ontario streams. Univ. Toronto Studies, Biol. Series 37, Publ. Ontario Fish. Res. Lab. $49: 114$ p.

Rivot E., Perrier C., Jouanin C., Roussel J.M., Riera P., Porcher J.-P. \& Baglinière J.-L., 2009. Climate Change may have Affected Growth and Life History in Atlantic Salmon Juveniles over the Past 30 Years. In: Haro et al. (Eds.), Challenges for diadromous fishes in a dynamic global environnement. American Fisheries Society Symposium 69 : 855-857.

Roche P.A., Billen G., Bravard J.-P., Décamps H., Pennequin D., Vindimian E. \& Wasson J.G., 2005. Les enjeux de recherche liés à la directive-cadre européenne sur l'eau. Comptes Rendus Geosciences 337(1-2) : 243-267. 
Rombough P.J., 1988. Respiratory gas exchange, aerobic metabolism and effects of hypoxia during early life. In: Hoar W.S. \& Randall D.J. (Ed.), Fish physiology 123-143.

Rosgen D., 1996. Applied river morphology. Wildland Hydrology Books. Colorado, $345 \mathrm{p}$.

Roussel J.M. \& Bardonnet A., 1999 - Ontogeny of diel pattern of stream margin habitat use by emerging brown trout (Salmo trutta L.) in experimental channels: influence of food and predator presence. Environmental Biology of Fish $56: 253-262$.

Rowe G. \& Wright G., 1999. The Delphi technique as a forecasting tool: issues and analysis. International Journal of Forecasting 15 : 353-375.

Sabaton C. \& Gras R., 1985. Étude des ressources piscicoles liées aux cours d'eau à débit réservé. Principes d'établissement des courbes de « préférence d'habitat ». Rapport EDF, Direction des Études et des Recherche $\mathrm{n}^{\circ}$ E31.85/03 : $12 \mathrm{p}$.

Souchon Y., Trocherie F., Fragnoud E. \& Lacombe C., 1989. Les modèles numériques des microhabitats des poissons: application et nouveaux développements. Revue des Sciences de l'Eau 2(4) : 807-830.

Southwood T.R.E., 1977. Habitat, the templet for ecological strategies? Journal of Animal Ecology 46(16) : 337-368.
Southwood T.R.E., 1988. Tactics, strategies and templets. Oikos 52 : 3-18.

Spaas J.T., 1958. Contribution to the comparative physiology and genetics of the european salmonidae. Hydrobiologia 11(3-4) : 267-274.

Strahler A.N., 1957. Quantitative analysis of watershed geomorphology. Transactions of the American Geophysical Union 38 : 913-920.

Valentin S., 1995. Variabilité artificielle des conditions d'habitat et conséquences sur les peuplements aquatiques : effets écologiques des éclusées hydroélectriques en rivière. Thèse de doctorat, $304 \mathrm{p}$.

Vannote R.L., Minshall W.M., Cummins K.W., Sedell J.R. \& Cushing C.E., 1980. The River Continuum Concept. Canadian Journal of Fisheries and Aquatic Sciences 37 : 130-137.

Verneaux J., 1973. Cours d'eau de FrancheComté (Massif du Jura). Recherches écologiques sur le réseau hydrographique du Doubs. Essai de biotypologie. Thèse d'état, $257 \mathrm{p}$.

Wasson J.G., 1989. Eléments pour une typologie fonctionnelle des eaux courantes. 1. Revue critique de quelques approches existantes. Bulletin d'Écologie 20(2) : 109-127. 